\title{
Trace anomaly and counterterms in designer gravity
}

\author{
Andrés Anabalón, ${ }^{a}$ Dumitru Astefanesei, ${ }^{b, c}$ David Choque ${ }^{c, d}$ and Cristián Martínez ${ }^{e}$ \\ ${ }^{a}$ Departamento de Ciencias, Facultad de Artes Liberales \\ and Facultad de Ingeniería y Ciencias, Universidad Adolfo Ibáñez, \\ Av. Padre Hurtado 750, Viña del Mar, Chile \\ ${ }^{b}$ Instituto de Física, Pontificia Universidad Católica de Valparaíso, \\ Casilla 4059, Valparaíso, Chile \\ ${ }^{c}$ Max-Planck-Institut für Gravitationsphysik, Albert-Einstein-Institut, \\ 14476 Golm, Germany

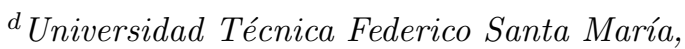 \\ Av. España 1680, Valparaíso, Chile \\ ${ }^{e}$ Centro de Estudios Científicos (CECs), \\ Av. Arturo Prat 514, Valdivia, Chile \\ E-mail: andres.anabalon@uai.cl, dumitru.astefanesei@ucv.cl, \\ brst1010123@gmail.com, martinez@cecs.cl
}

ABSTRACT: We construct concrete counterterms of the Balasubramanian-Kraus type for Einstein-scalar theories with designer gravity boundary conditions in $\mathrm{AdS}_{4}$, so that the total action is finite on-shell and satisfy a well defined variational principle. We focus on scalar fields with the conformal mass $m^{2}=-2 l^{-2}$ and show that the holographic mass matches the Hamiltonian mass for any boundary conditions. We compute the trace anomaly of the dual field theory in the generic case, as well as when there exist logarithmic branches of non-linear origin. As expected, the anomaly vanishes for the boundary conditions that are AdS invariant. When the anomaly does not vanish, the dual stress tensor describes a thermal gas with an equation of state related to the boundary conditions of the scalar field. In the case of a vanishing anomaly, we recover the dual theory of a massless thermal gas. As an application of the formalism, we consider a general family of exact hairy black hole solutions that, for some particular values of the parameters in the moduli potential, contains solutions of four-dimensional gauged $\mathcal{N}=8$ supergravity and its $\omega$-deformation. Using the AdS/CFT duality dictionary, they correspond to triple trace deformations of the dual field theory.

KEYwORDs: AdS-CFT Correspondence, Black Holes, Gauge-gravity correspondence

ARXIV EPRINT: 1511.08759 


\section{Contents}

1 Introduction 1

2 General $\mathrm{AdS}_{4}$ boundary conditions and multi-trace deformations in the dual theory

$\begin{array}{llr}3 & \text { Counterterms and regularized action } & 8\end{array}$

3.1 Variational principle 8

$\begin{array}{ll}3.2 & \text { Regularized action and free energy } \\ \end{array}$

4 Regularized Brown-York stress tensor $\quad 12$

5 Hamiltonian mass and holographic mass $\quad \mathbf{1 5}$

$\begin{array}{lll}5.1 & \text { Hamiltonian formalism } & 15\end{array}$

5.2 Non-logarithmic and logarithmic branches for $m^{2}=-2 / l^{2} \quad 17$

$\begin{array}{ll}5.3 \text { Holographic mass matches Hamiltonian mass } & 19\end{array}$

$\begin{array}{lll}5.4 & \text { Exact hairy solutions and triple-trace deformations } & 19\end{array}$

6 Conclusions 21

\section{Introduction}

A well-known check of the AdS/CFT duality [1] is the exact matching of the $\mathrm{AdS}_{5}$ vacuum energy and the Casimir energy of the large $N$ limit of $\mathrm{SU}(N), \mathcal{N}=4$ super Yang-Mills on $S^{3}[2,3]$. Technically speaking, this result was implemented by fixing the boundary data, namely imposing Dirichlet boundary conditions. Indeed, AdS is not globally hyperbolic, which means that besides initial conditions it is necessary to also provide boundary conditions for the evolution of a given field so that is well-defined. This was analyzed in-extenso for the linearized dynamics of spin-0, 1, and 2 fields in AdS [4], where, in particular, all the self-adjoint extensions for the relevant spin-0 Sturm-Liouville operators were found for scalar fields of mass $m$, which satisfy

$$
m_{\mathrm{BF}}^{2}+\frac{1}{l^{2}}>m^{2} \geq m_{\mathrm{BF}}^{2}, \quad m_{\mathrm{BF}}^{2}=-\frac{(D-1)^{2}}{4 l^{2}}
$$

where $l$ is the AdS radius, $D$ is the spacetime dimension, and $m_{\mathrm{BF}}^{2}$ is the BreitenlohnerFreedman (BF) bound [5, 6]. Furthermore, the backreaction of these generalized boundary conditions was considered, and its contribution to the spacetime energy has been computed in different ways [7-14].

One of the interesting outputs of [12] is the existence of logarithmic branches of nonlinear origin at certain values of the scalar field mass. In particular, this occurs when the 
scalar field mass is the one of the scalars of four-dimensional gauged $\mathcal{N}=8$ supergravity, namely $m^{2}=-2 l^{-2}$. It is interesting to note that the interpretation of the AdS invariant boundary conditions as a multi-trace deformation of the dual theory was given before this exhaustive analysis in [15] (see, also, the nice work [16]). Some of these theories are equivalently defined by the requirement of the existence of a soliton with a given value of the scalar field at the origin. Such constructions go by the name of designer gravity [11]. Techniques for constructing exact hairy black hole solutions that are relevant for our work can be found in [17-20].

In $[3,21,22]$, the energy of the gravitational configuration is connected to the gauge theory through the quasi-local Brown-York energy momentum tensor [23]. A non-trivial contribution to the mass when the boundary conditions break the conformal symmetry is expected to imply a modification of the trace anomaly, as was shown to be the case using the Hamilton-Jacobi equation in [24]. However, to the best of our knowledge, the same construction has not been done in the spirit of the work by Balasubramanian and Kraus [3], neither extended to the logarithmic branches of non-linear origin at $m^{2}=-2 l^{-2}$, which is the main objective of this paper. ${ }^{1}$ We use the Hamiltonian formalism as a guide for constructing the counterterms.

It is important to emphasize that, when the conformal symmetry is broken in the boundary, the mass of Ashtekar-Magnon-Das (AMD) [28, 29] does not match the Hamiltonian mass [14] and so it also does not match the holographic mass. Therefore, for AdS black hole solutions when the conformal symmetry is broken in the boundary, e.g [30], the right mass is not the AMD mass. As a concrete application, we shall consider a relatively simple family of hairy black hole solutions [31] that, however, is general enough to include the single scalar field truncations of four-dimensional gauged $\mathcal{N}=8$ supergravity as well as its $\omega$-deformation [32] (see, also, [33-35]).

The remainder of the paper is organized as follows: in section 2 we review mixed AdS boundary conditions in the context of AdS/CFT duality. In section 3 we provide the counterterms that regularize the action and verify that the variational principle is well defined when the action is supplemented with these counterterms. We compute the free energy of a generic hairy black hole solution with mixed boundary conditions of the scalar field and the relevant thermodynamical quantities. Section 4 contains the general formalism for computing the regularized quasilocal stress tensor (for both, the logarithmic and non-logarithmic branches). Using the AdS/CFT duality dictionary, we then compute the stress tensor of the dual field theory and the anomaly when the boundary conditions break conformal symmetry. In section 5 we compare different types of constructions of gravitational mass in AdS for mixed boundary conditions of the scalar field. When the conformal symmetry is broken the holographic and Hamiltonian mass match, but do not match the AMD mass. Then, we work out concrete examples of hairy black hole solutions that are dual to triple trace deformations of the boundary field theory. Finally, we end with some conclusions and future directions.

\footnotetext{
${ }^{1}$ In three dimensions, similar work was done in [25] and for the particular case of Dirichlet boundary conditions see, e.g., [26, 27].
} 


\section{General $\mathrm{AdS}_{4}$ boundary conditions and multi-trace deformations in the dual theory}

In this section, we review the role of AdS boundary conditions in the context of AdS/CFT duality [1]. According to 'holographic' dictionary, imposing mixed boundary conditions on the scalar field (in the bulk) corresponds to perturbing the large $N$ boundary theory by a relevant, irrelevant or marginal multi-trace deformation [15].

Let us start by exhibiting some known facts about the AdS/CFT duality [1]. We would like to describe what kind of boundary conditions preserve the conformal symmetry of the dual field theory and interpret them in the context of the AdS/CFT duality [10, 12, 15]. First, we describe the $\mathrm{AdS}_{4}$ spacetime and explain how the symmetries of the two dual theories match. That is, the isometry group $\mathrm{SO}(3,2)$ of $\mathrm{AdS}_{4}$ acts on the (conformal) boundary as the conformal group ${ }^{2}$ acting on Minkowski spacetime.

AdS spacetime has the maximal number of isometries in every dimension. Hence, it has a simple form in a large number of coordinate systems (see, e.g., [36] for a discussion in the context of AdS/CFT duality). Depending on the choice of the radial coordinate, the slices at constant radius can have a different geometry or even a different topology. For example, one can foliate $\mathrm{AdS}_{4}$ with the following slices:

$$
d \bar{s}^{2}=\bar{g}_{\mu \nu} d x^{\mu} d x^{\nu}=-\left(k+\frac{r^{2}}{l^{2}}\right) d t^{2}+\frac{d r^{2}}{k+\frac{r^{2}}{l^{2}}}+r^{2} d \Sigma_{k}^{2}
$$

where $k=\{+1,0,-1\}$ for the spherical $\left(d \Sigma_{1}^{2}=d \Omega^{2}\right)$, toroidal $\left(d \Sigma_{0}^{2}=d x^{2}+d y^{2}\right)$, and hyperbolic $\left(d \Sigma_{-1}=d H^{2}\right)$ foliations, respectively. Here, $d \Omega^{2}$ and $d H^{2}$ are the unit metrics on the 2-dimensional sphere and hyperboloid, respectively. The radius $l$ of $\mathrm{AdS}_{4}$ is related to the cosmological constant by $\Lambda=-3 / l^{2}$.

The conformal boundary is at $r \rightarrow \infty$, for which the induced metric is

$$
h_{a b} d x^{a} d x^{b}=\frac{r^{2}}{l^{2}}\left(-d t^{2}+l^{2} d \Sigma_{k}^{2}\right)
$$

and now it is clear that the background geometry where the field theory lives is related to the boundary geometry by a conformal transformation. Therefore, a bulk metric is associated with a conformal structure at infinity. The conformal factor is going to play an important role when we are going to compute the boundary stress tensor.

Even if different foliations of $\mathrm{AdS}_{4}$ are related by local coordinate transformations, the corresponding dual gauge theories are physically inequivalent (for example, in the $k=1$ case there is a Hawking-Page phase transition, but not for $k=0$ ). This is due to the fact that different spacelike foliations of the background geometry lead to different definitions of the time coordinate (and so the Hamiltonian) of the dual quantum system.

Starting with $k=0$ form of $\mathrm{AdS}_{4}$ metric and using the change of coordinates $r=l^{2} / z$, we obtain

$$
d \bar{s}^{2}=\frac{l^{2}}{z^{2}}\left(d z^{2}-d t^{2}+d x^{2}+d y^{2}\right)
$$

\footnotetext{
${ }^{2}$ The conformal group of Minkowski spacetime is the invariance group of the light cone, in other words all the transformations that leave $d s^{2}=0$ invariant.
} 
In these coordinates, which cover only part of $\mathrm{AdS}_{4}$ spacetime, the Minkowski spacetime appears naturally as the conformal boundary. The finite isometries of $\mathrm{AdS}_{4}$ map the boundary $z=0$ to itself and, moreover, act as conformal transformations in the boundary. In particular, the transformation $(z, t, x, y) \leftarrow \lambda(z, t, x, y)$, which leaves the metric (2.3) invariant, acts as the dilation (scale transformation) in the boundary. Since the AdS spacetime is not globally hyperbolic, one has to impose boundary conditions. Within the AdS/CFT duality, various deformations of the AdS boundary conditions are interpreted as dual to deformations of the CFT. It is well known [4-6] that a scalar of arbitrary mass in AdS can have both normalizable and non-normalizable modes. It was shown in $[37,38]$ that the normalizable modes describe fluctuations in the bulk and the non-normalizable modes correspond to operator insertions in the boundary dual field theory. We are interested in the case when both modes are normalizable:

$$
m_{\mathrm{BF}}^{2}+\frac{1}{l^{2}}>m^{2} \geq m_{\mathrm{BF}}^{2}, \quad m_{\mathrm{BF}}^{2}=-\frac{9}{4 l^{2}}
$$

where $m_{\mathrm{BF}}^{2}$ is the BF bound (1.1) in four dimensions.

In what follows we briefly review the boundary conditions that accommodate a scalar field whose mass corresponds to the conformal one. We are interested in the action

$$
I\left[g_{\mu \nu}, \phi\right]=\int_{\mathcal{M}} d^{4} x \sqrt{-g}\left[\frac{R}{2 \kappa}-\frac{1}{2}(\partial \phi)^{2}-V(\phi)\right]+\frac{1}{\kappa} \int_{\partial \mathcal{M}} d^{3} x K \sqrt{-h}
$$

where $V(\phi)$ is the scalar potential, $\kappa=8 \pi G$ with $G$ the Newton gravitational constant, and the last term is the Gibbons-Hawking boundary term. Here, $h$ is the determinant of the boundary metric and $K$ is the trace of the extrinsic curvature. The equations of motion for the scalar field and metric are

$$
\begin{aligned}
& \frac{1}{\sqrt{-g}} \partial_{\mu}\left(\sqrt{-g} g^{\mu \nu} \partial_{\nu} \phi\right)-\frac{\partial V}{\partial \phi}=0 \\
& E_{\mu \nu}=R_{\mu \nu}-\frac{1}{2} g_{\mu \nu} R-\kappa T_{\mu \nu}^{\phi}=0
\end{aligned}
$$

where the stress tensor of the scalar field is

$$
T_{\mu \nu}^{\phi}=\partial_{\mu} \phi \partial_{\nu} \phi-g_{\mu \nu}\left[\frac{1}{2}(\partial \phi)^{2}+V(\phi)\right]
$$

We work with the general ansatz

$$
d s^{2}=-N(r) d t^{2}+H(r) d r^{2}+S(r) d \Sigma_{k}^{2}
$$

As it was shown first in three dimensions [7], and then generalized in four and higher dimensions $[9,10,12,13]$, in the presence of the scalar fields the standard AdS boundary conditions are modified. One can obtain the right fall-off for the $g_{r r}$ component of the metric by considering the equations of motion and using the fall-off of the scalar field. A general discussion for any mass of the scalar field in the range (1.1) can be found in [12], 
but in this work we focus on the concrete case of the conformal mass in four dimensions $m^{2}=-2 l^{-2}$. We start with the potential

$$
V(\phi)=-\frac{3}{\kappa l^{2}}-\frac{\phi^{2}}{l^{2}}+O\left(\phi^{4}\right)
$$

The fall-off of the scalar field in this case is

$$
\phi(r)=\frac{\alpha}{r}+\frac{\beta}{r^{2}}+O\left(r^{-3}\right)
$$

In order to accommodate the black hole of section 5.4 we consider the following asymptotic behavior for the $N(r)$ and $S(r)$ metric coefficients

$$
\begin{aligned}
N(r) & =-g_{t t}=\frac{r^{2}}{l^{2}}+k-\frac{\mu}{r}+O\left(r^{-2}\right) \\
S(r) & =r^{2}+O\left(r^{-2}\right)
\end{aligned}
$$

Now, we use the combination of the equations of motion $(2.7), E_{t}^{t}-E_{r}^{r}=0$, from which we obtain

$$
N S^{\prime 2} H-2 N S^{\prime \prime} H S+(N H)^{\prime} S^{\prime} S-2 \kappa N H S^{2} \phi^{\prime 2}=0
$$

and then

$$
H(r)=g_{r r}=\frac{l^{2}}{r^{2}}+\frac{l^{4}}{r^{4}}\left(-k-\frac{\alpha^{2} \kappa}{2 l^{2}}\right)+\frac{l^{5}}{r^{5}}\left(\frac{\mu}{l}-\frac{4 \kappa \alpha \beta}{3 l^{3}}\right)+O\left(r^{-6}\right)
$$

The reason we would like to obtain the fall-off of $g_{r r}$ in this way is because the Hamiltonian mass can be read off from it - if there is a contribution of the scalar field to the mass, one should be able to identify it in $g_{r r}$.

From now on, we use the generic notation for the expansion of $g_{r r}$ as

$$
g_{r r}=\frac{l^{2}}{r^{2}}+\frac{a l^{4}}{r^{4}}+\frac{b l^{5}}{r^{5}}+O\left(r^{-6}\right)
$$

where $a=-k-\frac{\kappa \alpha^{2}}{2 l^{2}}$ and $b=\frac{\mu}{l}-\frac{4 \kappa \alpha \beta}{3 l^{3}}$. At this point, it is interesting to investigate when the asymptotic conditions are AdS invariant and the Hamiltonian is well defined. It seems that, for some special functional relationship on the coefficients $\alpha$ and $\beta$ of the modes of the scalar field, both conditions are satisfied. This was explicitly done in $[10,12]$ and here we just present the result:

$$
\beta=C \alpha^{2}
$$

Interestingly enough, one can also obtain a finite Hamiltonian when the boundary conformal symmetry is broken.

A similar analysis can be done for the so-called logarithmic branch [9]. In what follows we would like to carefully analyze this case and present details we are going to use in the next sections.

It is well known that a second order differential equation has two linearly independent solutions. When the ratio of the roots of the indicial equation is an integer, the solution may develop a logarithmic branch. This is exactly what happens when the scalar field 
saturates the BF bound, in which case the leading fall-off contains a logarithmic term [9]. However, we are interested in a scalar field with the conformal mass $m^{2}=-2 l^{-2}$. To obtain the logarithmic branch, a cubic term in the asymptotic expansion of the scalar field potential is necessary [12]

$$
V(\phi)=-\frac{3}{\kappa l^{2}}-\frac{\phi^{2}}{l^{2}}+\lambda \phi^{3}+O\left(\phi^{4}\right)
$$

so that the fall-off of the scalar field to be considered is

$$
\phi(r)=\frac{\alpha}{r}+\frac{\beta}{r^{2}}+\frac{\gamma \ln (r)}{r^{2}}+O\left(r^{-3}\right)
$$

To obtain the fall-off of $g_{r r}$ we use the same fall-off for the other components of the metric and the same combination of the equations of motion as in the non-logarithmic branch, $E_{t}^{t}-E_{r}^{r}=0$. We get

$$
H(r)=g_{r r}=\frac{l^{2}}{r^{2}}+\frac{l^{4}}{r^{4}}\left(-k-\frac{\kappa \alpha^{2}}{2 l^{2}}\right)+\frac{l^{5}}{r^{5}}\left(\frac{\mu}{l}-\frac{4 \kappa \alpha \beta}{3 l^{3}}+\frac{2 \kappa \alpha \gamma}{9 l^{3}}\right)+\frac{l^{5} \ln r}{r^{5}}\left(-\frac{4 \kappa \alpha \gamma}{3 l^{3}}\right)+O\left[\frac{\ln (r)^{2}}{r^{6}}\right]
$$

Using again the generic notation for the asymptotic expansion of $g_{r r}$

$$
H(r)=\frac{l^{2}}{r^{2}}+\frac{l^{4} a}{r^{4}}+\frac{l^{5} b}{r^{5}}+\frac{l^{5} c \ln r}{r^{5}}+O\left[\frac{\ln (r)^{2}}{r^{6}}\right]
$$

we identify the relevant coefficients as

$$
a=-k-\frac{\alpha^{2} \kappa}{2 l^{2}} ; \quad b=\frac{\mu}{l}-\frac{4 \kappa \alpha \beta}{3 l^{3}}+\frac{2 \kappa \alpha \gamma}{9 l^{3}} ; \quad c=-\frac{4 \kappa \gamma \alpha}{3 l^{3}}
$$

Now, let us check when the fall-off of the scalar field we have considered is compatible with its equation of motion:

$$
\partial_{r}\left(\frac{\phi^{\prime} S \sqrt{N}}{\sqrt{H}}\right)-S \sqrt{N H} \frac{\partial V}{\partial \phi}=0
$$

In the asymptotic region, $r \rightarrow \infty$, this equation becomes

$$
\frac{3 \alpha^{2} l^{2} \lambda+\gamma}{l^{2}}+O\left(r^{-1}\right)=0
$$

and so the coefficient $\gamma$ is fixed by $\alpha$ as $\gamma=-3 l^{2} \lambda \alpha^{2}$ (or, using the notation that we shall use below, $\gamma=C_{\gamma} \alpha^{2}$, where $C_{\gamma}=-3 l^{2} \lambda$ ). This result is important because, as we will see shortly, is also part of the conditions that preserve the conformal symmetry of the boundary.

The last step in our derivation is to investigate when the boundary conditions are preserved under the asymptotic AdS symmetry. The corresponding asymptotic Killing vector $\xi^{\mu}=\left(\xi^{r}, \xi^{m}\right)$ is

$$
\begin{aligned}
\xi^{r} & =r \eta^{r}\left(x^{m}\right)+O\left(r^{-1}\right) \\
\xi^{m} & =O(1)
\end{aligned}
$$


where $\{m\}$ is an index that run over the time an angular coordinates. The fall-off of the scalar field should be invariant under the asymptotic AdS symmetries and so we obtain:

$$
\phi^{\prime}(x)=\phi(x)+\xi^{\mu} \partial_{\mu} \phi(x)=\frac{\bar{\alpha}}{r}+\frac{\bar{\beta}}{r^{2}}+\frac{\bar{\gamma} \ln (r)}{r^{2}}+O\left(r^{-3}\right)
$$

where

$$
\begin{aligned}
& \bar{\alpha}=\alpha-\eta^{r} \alpha+\xi^{m} \partial_{m} \alpha \\
& \bar{\beta}=\beta-\eta^{r}(2 \beta-\gamma)+\xi^{m} \partial_{m} \beta \\
& \bar{\gamma}=\gamma-2 \gamma \eta^{r}+\xi^{m} \partial_{m} \gamma
\end{aligned}
$$

If the coefficients in the series (2.26) are functionally related, the conformal symmetry on the boundary fixes the functional relation between the coefficients so that the equations above are compatible. Hence, one performs a Taylor expansion of $\bar{\gamma}$ and $\bar{\beta}$ to linear order in $\eta^{r}$ and $\xi^{m}$ to obtain:

$$
\eta^{r}\left(2 \gamma-\alpha \frac{\partial \gamma}{\partial \alpha}\right)+\xi^{m}\left(\frac{\partial \alpha}{\partial x^{m}} \frac{\partial \gamma}{\partial \alpha}-\frac{\partial \gamma}{\partial x^{m}}\right)=0
$$

and

$$
\eta^{r}\left(2 \beta-\gamma-\alpha \frac{\partial \beta}{\partial \alpha}\right)+\xi^{m}\left(\frac{\partial \alpha}{\partial x^{m}} \frac{\partial \beta}{\partial \alpha}-\frac{\partial \beta}{\partial x^{m}}\right)=0 .
$$

Using the fact that $\eta^{r}$ and $\xi^{m}$ are independent, we get from (2.28) that $2 \gamma=\alpha \frac{\partial \gamma}{\partial \alpha}$, which implies that $\gamma=C_{\gamma} \alpha^{2}$. This is the result obtained before from the equation of motion for the scalar field. From the integration of (2.29) we obtain

$$
\beta(\alpha)=\left(-C_{\gamma} \ln (\alpha)+C\right) \alpha^{2}
$$

When $C_{\gamma}=0$ this result matches the condition found for the non-logarithmic branch (2.17). Again, one can obtain a finite Hamiltonian even if the conformal invariance is broken.

A precise formulation of the AdS/CFT duality [1] was proposed in [39, 40] and developed for multi-trace deformations in [15]. The observables in the field theory side of the duality are the correlation functions of gauge invariant operators, which are composites of the elementary fields. Any supergravity field $\phi$ corresponds to an operator $O$ in the (boundary) field theory. The duality relates the generating functional for correlation functions of the operator $O$ with the string/gravity partition function on AdS space with the boundary conditions that are imposed on the excitations in the bulk. In our case, the relevant fields in the bulk are the graviton (metric perturbations) and scalar field. The corresponding operators in the dual field theory are the stress-energy tensor $T_{\mu \nu}$ of the dual field theory and a scalar operator of dimension $\Delta$, respectively.

Let us consider a massive scalar field. By solving the equation of motion close to the boundary, we obtain:

$$
\phi(r)=\frac{\alpha}{r^{\Delta_{-}}}+\frac{\beta}{r^{\Delta_{+}}}+\ldots
$$

where $\alpha$ and $\beta$ are the leading and sub-leading components of the asymptotic expansion of the scalar field and $\Delta_{ \pm}=\frac{3}{2} \pm \sqrt{\frac{9}{4}+m^{2} l^{2}}$. 
Depending of the value of the mass, the two modes (on the Lorentzian section) can be divergent or finite. For example, for a positive squared-mass $m^{2}>0$ the mode $\beta$ is divergent in the interior and finite at the boundary and the mode $\alpha$ is divergent at the boundary but finite in the interior. Then, the mode $\beta$ corresponds to source currents in the boundary dual theory. On the other hand, by turning on the mode $\alpha$, the bulk geometry is modified while the AdS structure near the boundary may be preserved - this is the type of deformation we are interested in this work. Since the bulk gravity solution is changed, one has to perform a linearized analysis around the new background to calculate the correlation functions. This is exactly what happens when the 'vacuum' around which one expands to obtain the physical quantities is changed. Then, in the dual theory, there is a similar situation: the dual field theory is expanded around a vacuum with non-trivial vacuum expectations values (VEV) for the appropriate operators. Indeed, in the standard AdS/CFT dictionary [37, 38], a bulk gravity solution with a non-trivial dilaton corresponds in the dual field theory to the insertion of a source for an operator with conformal dimension $\Delta_{-}, \operatorname{VEV} \alpha$, and current $\beta=J(x)$.

The spectrum of operators in the dual field theory include all the gauge invariant quantities, namely product of traces of products of fields (or the sum of such products). Single-trace operators in the field theory may be identified with single-particle states in AdS, while multiple-trace operators correspond to multi-particle states. The significance of the multi-trace deformations from a point of view of the gravity side was investigated in $[10,15]$. The mixed boundary conditions play an important role because they correspond to a deformation of the field theory action by

$$
I_{\mathrm{CFT}} \rightarrow I_{\mathrm{CFT}}-\int d^{3} x W[\mathcal{O}(x)]
$$

where $\beta(x)=\frac{d W}{d \alpha(x)}$, and $W$ is fixed by the boundary conditions of the string theory side.

In section 5 we are going to apply this general framework to concrete analytic hairy black hole solutions.

\section{Counterterms and regularized action}

The usual approach to computing thermodynamic quantities of black holes is to analytically continue in the time coordinate in order to obtain a Euclidean solution of the Einstein equations (with negative cosmological constant). In this way, the periodicity of the Euclidean time is related to the temperature of the black hole and the Euclidean action to the thermodynamical potential (in our case, the free energy). In this section we explicitly construct counterterms that cancel the divergences of the action for both logarithmic and non-logarithmic branches and check that the variational principle is well possed. We apply the counterterm method to compute the free energy of hairy black holes with a scalar field with the conformal mass $m^{2}=-2 l^{-2}$.

\subsection{Variational principle}

Our goal is to construct counterterms (boundary terms) that regularize the action so that the variational principle is well-posed. The boundary terms do not change the equations 
of motion and so they can be incorporated in the action. To make our point, let us first consider the action (2.5) when the scalar field is turned off. In this case, the action has just two terms: the bulk action and the Gibbons-Hawking surface term necessary to ensure that the Euler-Lagrange variation is well-defined. The gravitational action computed in this way (even at tree-level) contains divergences that arise from integrating over the infinite volume of spacetime. In the AdS/CFT context, the infrared (IR) divergences of gravity are interpreted as ultraviolet (UV) divergences of the dual CFT. A well understood way of computing the bulk action without introducing a background is to add local counterterms into the action, which remove all divergences, leading to a finite action corresponding to the partition function of the CFT. For pure AdS gravity in four dimensions, the action should be supplemented with the following counterterm [3]:

$$
I_{g}^{c t}=-\frac{1}{\kappa} \int_{\partial \mathcal{M}} d^{3} x \sqrt{-h}\left(\frac{2}{l}+\frac{\mathcal{R} l}{2}\right)
$$

Here, $h_{a b}$ is the induced metric on the boundary and $\mathcal{R}$ is its Ricci scalar.

In the presence of the scalar field, this counterterm is not sufficient to cancel the divergences in the action. For this case, an additional boundary term that depends on the scalar is needed, namely $I_{\phi}$. We are going to study the variational principle of the following action:

$I=\int d^{4} x \sqrt{-g}\left(\frac{R}{2 \kappa}-\frac{(\partial \phi)^{2}}{2}-V(\phi)\right)+\frac{1}{\kappa} \int_{\partial \mathcal{M}} d^{3} x \sqrt{-h} K-\frac{1}{\kappa} \int_{\partial \mathcal{M}} d^{3} x \sqrt{-h}\left(\frac{2}{l}+\frac{\mathcal{R} l}{2}\right)+I_{\phi}^{c t}$

for a scalar field with the conformal mass $m=-2 l^{-2}$. In some previous work (for example, see $[41,42])$, the following counterterm that produces a finite action for the non-logarithmic branch was proposed:

$$
\frac{1}{6 \kappa} \int_{\partial \mathcal{M}} d^{3} x \sqrt{-h}\left(\phi n^{\nu} \partial_{\nu} \phi-\frac{\phi^{2}}{2 l}\right)
$$

However, it is problematic because it is not intrinsic to the boundary and also, for mixed boundary conditions, the variational principle is not satisfied. Instead, we propose new counterterms for both, the logarithmic and non-logarithmic branches, so that the action is finite and there is a well-posed variational principle. These intrinsic counterterms are constructed to be compatible with the Hamiltonian method in the sense that the results match for any boundary conditions.

Let us start with the non-logarithmic branch with the boundary term associated to the scalar field given by

$$
I_{\phi}^{c t}=-\int_{\partial \mathcal{M}} d^{3} x \sqrt{-h}\left[\frac{\phi^{2}}{2 l}+\frac{W(\alpha)}{l \alpha^{3}} \phi^{3}\right]
$$

Then, by using the boundary expansion of the metric and scalar field, the variation of the action yields a boundary term evaluated at the cutoff $r$ :

$$
\delta I=\int d^{3} x \sqrt{-h}\left[\frac{1}{r}\left(-\sqrt{g^{r r}} \phi^{\prime}-\frac{\phi}{l}-\frac{3 W(\alpha) \phi^{2}}{l \alpha^{3}}\right)\left(1+\frac{1}{r} \frac{d^{2} W(\alpha)}{d \alpha^{2}}\right)+\left(\frac{3 W(\alpha)}{\alpha}-\beta\right) \frac{\phi^{3}}{l \alpha^{3}}\right] \delta \alpha
$$


It is easy to show then that the variational principle is well defined when the cutoff goes to infinity:

$$
\lim _{r \rightarrow \infty} \delta I=0
$$

For the logarithmic branch we should work with the following counterterm for the scalar field:

$$
I_{\phi}^{c t}+\bar{I}_{\phi}^{c t}=-\int_{\partial \mathcal{M}} d^{3} x \sqrt{-h}\left[\frac{\phi^{2}}{2 l}+\frac{\phi^{3}}{l \alpha^{3}}\left(W-\frac{\alpha \gamma}{3}\right)-\frac{\phi^{3} C_{\gamma}}{3 l} \ln \left(\frac{\phi}{\alpha}\right)\right]
$$

where $\bar{I}_{\phi}^{c t}$ is the counterterm necessary to provide a well possed acction principal in the case of the logartithmic branch. Using the asymptotic expansions of the metric and scalar field we also obtain that the variational principle is well-defined for arbitrary boundary conditions when the cut-off surface is send to infinity. As we shall show below, the same counterterms provide a finite on-shell action and the right free energy.

\subsection{Regularized action and free energy}

Evaluating the action leads to a formally divergent result. Now, we would like to show that, indeed, all the divergences can be eliminated by using the counterterms proposed in the previous section and so the action is finite. We use the standard technique of Wick rotating the time direction $t=i \tau$. Then, the temperature is related to the periodicity of the Euclidean time $\tau(\Delta \tau=\beta=1 / T)$ and the leading contribution to the free energy is determined by evaluating the Euclidean action.

The action has four terms, the bulk part $I_{\text {bulk }}^{E}$, Gibbons-Hawking surface term $I_{G H}^{E}$, and two boundary counterterms $\left(I_{g}^{c t}, I_{\phi}^{c t}\right): I=I_{\text {bulk }}^{E}+I_{G H}^{E}+I_{g}^{c t}+I_{\phi}^{c t}$. Let us compute these contributions for the non-logarithmic branch first.

Since we are going to study the properties of a large family of exact hairy black hole solutions, let us start with the following generic metric ansatz

$$
d s^{2}=\Omega(x)\left[-f(x) d t^{2}+\frac{\eta^{2} d x^{2}}{f(x)}+d \Sigma_{k}^{2}\right]
$$

Concrete expressions for the functions $\Omega(x)$ and $f(x)$ are presented in section 5.4.

The computations in the $(t, x, \Sigma)$ coordinate system (3.8) are related by a simple coordinate transformation to $(t, r, \Sigma)$ system (2.9). In what follows, $x_{b}$ and $r_{b}$ denote the boundary, and $x_{+}$and $r_{+}$the horizon. The on-shell Euclidean bulk action can be written as

$$
I_{\mathrm{bulk}}^{E}=\int_{0}^{1 / T} d \tau \int_{x_{+}}^{x_{b}} d^{3} x \sqrt{g^{E}} V(\phi)=\left.\frac{\sigma_{k}}{2 \eta \kappa T} \frac{d(\Omega f)}{d x}\right|_{x_{+}} ^{x_{b}}
$$

where $\sigma_{k}$ is the area of $\Sigma_{k}$ (e.g., for $k=1 \sigma_{1}=4 \pi$ ) and $g^{E}$ is the metric on the Euclidean section. The two coordinate systems $\left(t, x, \Sigma_{k}\right)$ and $\left(t, r, \Sigma_{k}\right)$ are related by

$$
\Omega(x) \rightarrow S(r) ; \quad f(x) \rightarrow \frac{N(r)}{S(r)} ; \quad d x \rightarrow \frac{\sqrt{N H}}{\eta S} d r
$$

and so we can rewrite the bulk integral result in the coordinates $\left(t, r, \Sigma_{k}\right)$ as

$$
I_{\text {bulk }}^{E}=\left.\frac{\sigma_{k}}{2 \kappa T} \frac{S}{\sqrt{N H}} \frac{d N}{d r}\right|_{r_{+}} ^{r_{b}}
$$


Let us now compute the Gibbons-Hawking term. Consider a timelike hypersurface $x=x_{0}$, then the induced metric $h^{\mu \nu}=g^{\mu \nu}-n^{\mu} n^{\nu}$, normal, extrinsic curvature, and its trace $K=h^{\mu \nu} K_{\mu \nu}$ are

$$
\begin{aligned}
& d s^{2}=h_{a b} d x^{a} d x^{b}=\Omega\left(x_{0}\right)\left[-f\left(x_{0}\right) d t^{2}+d \Sigma_{k}\right] \\
& n_{a}=\left.\frac{\delta_{a}^{x}}{\sqrt{g^{x x}}}\right|_{x=x_{0}} ; \quad K_{a b}=\left.\frac{\sqrt{g^{x x}}}{2} \partial_{x} g_{a b}\right|_{x=x_{0}} ; \quad K=\left.\frac{1}{2 \eta}\left(\frac{f}{\Omega}\right)^{1 / 2}\left[\frac{(\Omega f)^{\prime}}{\Omega f}+\frac{2 \Omega^{\prime}}{\Omega}\right]\right|_{x_{0}}
\end{aligned}
$$

and using the transformation equations (3.10) the contribution of this term can be rewritten as

$$
I_{G H}^{E}=-\left.\frac{\sigma_{k}}{\kappa T} \frac{\Omega f}{2 \eta}\left[\frac{(\Omega f)^{\prime}}{\Omega f}+\frac{2 \Omega^{\prime}}{\Omega}\right]\right|_{x_{b}}=-\left.\frac{\sigma_{k}}{2 T \kappa}\left(\frac{S}{\sqrt{N H}} \frac{d N}{d r}+\frac{2 N}{\sqrt{N H}} \frac{d S}{d r}\right)\right|_{r_{b}}
$$

The contribution from the gravitational counterterm is

$$
I_{g}^{c t}=\left.\frac{2 \sigma_{k}}{\kappa T l}\left(\Omega^{3 / 2} f^{1 / 2}+\frac{l^{2} k}{2} f^{1 / 2} \Omega^{1 / 2}\right)\right|_{x_{b}}=\left.\frac{2 \sigma_{k}}{\kappa T l} S \sqrt{N}\left(1+\frac{l^{2} k}{2 S}\right)\right|_{r_{b}}
$$

Using the general formula for the temperature

$$
T=\left.\frac{N^{\prime}}{4 \pi \sqrt{N H}}\right|_{r_{+}}
$$

one can write the sum of these three contributions in the total action as

$$
I_{\mathrm{bulk}}^{E}+I_{G H}^{E}+I_{g}^{c t}=-\frac{1}{T}\left[\frac{\sigma_{k} S\left(r_{+}\right) T}{4 G}\right]-\left.\frac{\sigma_{k}}{2 \kappa T}\left[\frac{2 N}{\sqrt{N H}} \frac{d S}{d r}-\frac{4}{l} S \sqrt{N}\left(1+\frac{l^{2} k}{2 S}\right)\right]\right|_{r_{b}}
$$

which, for a scalar field with the conformal mass $m^{2}=-2 l^{-2}$ as in (2.11) and with the metric fall-off (2.12) and (2.16), becomes

$$
I_{\mathrm{bulk}}^{E}+I_{G H}^{E}+I_{g}^{c t}=-\frac{\mathcal{A}}{4 G}-\left.\frac{\sigma_{k}}{T}\left(-\frac{\mu}{\kappa}+\frac{4 \alpha \beta}{3 l^{2}}+\frac{r \alpha^{2}}{2 l^{2}}\right)\right|_{r_{b}}
$$

Here, $\mathcal{A}=\sigma_{k} S\left(r_{+}\right)$is the horizon area.

It is clear now that the gravitational counterterm is not sufficient to remove the divergences at the boundary $r_{b} \rightarrow \infty$, but this new linear divergence can be regularized with the following counterterm that depends on the scalar field:

$$
I_{\phi}^{c t}=\int_{\partial \mathcal{M}} d^{3} x \sqrt{h^{E}}\left[\frac{\phi^{2}}{2 l}+\frac{W(\alpha)}{l \alpha^{3}} \phi^{3}\right]=\left.\frac{\sigma_{k}}{T}\left(\frac{W}{l^{2}}+\frac{\alpha \beta}{l^{2}}+\frac{r \alpha^{2}}{2 l^{2}}\right)\right|_{r_{\infty}}
$$

The renormalized Euclidean action can be rewritten then using $\beta=d W / d \alpha$ as

$$
I^{E}=I_{\mathrm{bulk}}^{E}+I_{G H}^{E}+I_{g}^{c t}+I_{\phi}^{c t}=-\frac{\mathcal{A}}{4 G}+\frac{\sigma_{k}}{T}\left[\frac{\mu}{\kappa}+\frac{1}{l^{2}}\left(W-\frac{\alpha}{3} \frac{d W}{d \alpha}\right)\right]
$$


and so the free energy becomes

$$
F=I^{E} T=M-T S
$$

The thermodynamic relations will provide the same mass and entropy for the black holes:

$$
M=-T^{2} \frac{\partial I^{E}}{\partial T}=\sigma_{k}\left[\frac{\mu}{\kappa}+\frac{1}{l^{2}}\left(W-\frac{\alpha}{3} \frac{d W}{d \alpha}\right)\right]
$$

and

$$
S=-\frac{\partial\left(I^{E} T\right)}{\partial T}=\frac{\mathcal{A}}{4 G}
$$

A similar computation can be done for the logarithmic branch. We work again with a scalar field with the conformal mass $m^{2}=-2 l^{-2}$ with a fall-off (2.19) for which the metric fall-off is (2.20). If we work with the counterterm (3.4), we obtain

$$
I_{\mathrm{bulk}}^{E}+I_{G H}^{E}+I_{g}^{c t}+I_{\phi}^{c t}=-\frac{\mathcal{A}}{4 G}+\frac{\sigma_{k}}{T}\left\{\frac{\mu}{\kappa}+\frac{1}{l^{2}}\left[W(\alpha)-\frac{\alpha}{3} \frac{d W}{d \alpha}+\frac{2 \alpha \gamma}{9}-\frac{\alpha \gamma}{3} \ln r\right]\right\}
$$

and we see that there is still a logarithmic divergence. Therefore, one consider a new contribution from the scalar field that will also cancel that divergence (3.7):

$$
\bar{I}_{\phi}^{c t}=\int_{\partial \mathcal{M}} d^{3} x \sqrt{h^{E}}\left\{\frac{\phi^{3} \gamma}{3 \alpha^{2} l}\left[\ln \left(\frac{\alpha}{\phi}\right)-1\right]\right\}=\frac{\sigma_{k}}{T}\left[-\frac{\alpha \gamma}{3 l^{2}}+\frac{\alpha \gamma \ln r}{3 l^{2}}+O\left(r^{-1} \ln r\right)\right]
$$

We also obtain a finite action for the logarithmic branch

$$
I^{E}=I_{\mathrm{bulk}}^{E}+I_{G H}^{E}+I_{g}^{c t}+I_{\phi}^{c t}+\bar{I}_{\phi}^{c t}=-\frac{\mathcal{A}}{4 G}+\frac{\sigma_{k}}{T}\left[\frac{\mu}{\kappa}+\frac{1}{l^{2}}\left(W-\frac{\alpha}{3} \frac{d W}{d \alpha}-\frac{\alpha \gamma}{9}\right)\right]
$$

where $\gamma=C_{\gamma} \alpha^{2}$ and $C_{\gamma}=-3 l^{2} \lambda$.

The thermodynamic relations provide the right results:

$$
M=-T^{2} \frac{\partial I^{E}}{\partial T}=\sigma_{k}\left[\frac{\mu}{\kappa}+\frac{1}{l^{2}}\left(W-\frac{\alpha}{3} \frac{d W}{d \alpha}-\frac{\alpha \gamma}{9}\right)\right]
$$

and

$$
S=-\frac{\partial\left(I^{E} T\right)}{\partial T}=\frac{\mathcal{A}}{4 G}
$$

The conformal symmetry of the boundary is preserved when $W(\alpha)=\alpha^{3}\left(C+l^{2} \lambda \ln \alpha\right)$.

\section{Regularized Brown-York stress tensor}

Within the AdS/CFT duality, the AdS graviton couples to the stress-energy tensor of the CFT [43, 44]:

$$
\int_{\partial \mathcal{M}} d^{3} x h^{a b} T_{a b}
$$

Then, from a holographic point of view, the Brown-York stress tensor is interpreted as the stress-energy tensor of the dual field theory. In this section we work in the coordinates 
$(t, r, \Sigma)_{k}$ for which the metric was given in (2.9). For the bulk geometry we use the foliation with the surfaces $r=R=$ constant and the induced metric is

$$
d s^{2}=h_{a b} d x^{a} d x^{b}=-N(R) d t^{2}+S(R) d \Sigma_{k}^{2}
$$

The Brown-York (quasilocal) stress tensor is defined as [23]

$$
\tau^{a b} \equiv \frac{2}{\sqrt{-h}} \frac{\delta I}{\delta h_{a b}}
$$

where $I$ is the total action including the counterterms.

Since the metric where the dual field lives is related to the boundary metric by a conformal factor, it is very important to emphasize that the CFT stress tensor is also related to the Brown-York stress tensor up to a conformal factor. As an warm up exercise, let us describe this method for the 4-dimensional Schwarzschild-AdS black hole - we are going to follow the analysis of [45].

The black hole metric is

$$
d s^{2}=-\left(1-\frac{m}{r}+\frac{r^{2}}{l^{2}}\right) d t^{2}+\left(1-\frac{m}{r}+\frac{r^{2}}{l^{2}}\right)^{-1} d r^{2}+r^{2} d \Omega^{2}
$$

and if we consider the foliation $r=R$ the induced metric $h_{a b}$ of any 'slice' is

$$
d s^{2}=-\left(1-\frac{m}{R}+\frac{R^{2}}{l^{2}}\right) d t^{2}+R^{2} d \Omega^{2}
$$

As we have pointed out before, the boundary metric is

$$
d s_{\text {boundary }}^{2}=\frac{R^{2}}{l^{2}}\left(-d t^{2}+l^{2} d \Omega^{2}\right)
$$

but the background metric where the dual quantum field theory lives is $\gamma_{a b}$ defined as

$$
d s_{\text {dual }}^{2}=\gamma_{a b} d x^{a} d x^{b}=-d t^{2}+l^{2} d \Omega^{2}
$$

The metric $\gamma_{a b}$ is not dynamical and it is related by a conformal factor to the boundary metric. The corresponding dual stress tensor is

$$
\left\langle\tau_{a b}^{\text {dual }}\right\rangle=\lim _{R \rightarrow \infty} \frac{R}{l} \tau_{a b}=\frac{m}{16 \pi G l^{2}}\left[3 \delta_{a}^{0} \delta_{b}^{0}+\gamma_{a b}\right]
$$

Written in this way [45] it has the form of a thermal gas of a massless particles and, as expected, its trace vanishes $\left\langle\tau^{\text {dual }}\right\rangle=\left\langle\tau_{a b}^{\text {dual }}\right\rangle \gamma^{a b}=0$.

A similar procedure can be used for the hairy black holes, but one should add the boundary counterterms related to the scalar field. In the case of the non-logarithmic branch, the complete action is (3.2) and the scalar counterterm was given in (3.3), where $G_{a b}$ is the Einstein tensor for the foliation (4.2) given by $G_{a b}=\delta_{a}^{t} \delta_{b}^{t} N k / S$. The regularized stress tensor is

$$
\tau_{a b}=-\frac{1}{\kappa}\left(K_{a b}-h_{a b} K+\frac{2}{l} h_{a b}-l G_{a b}\right)-\frac{h_{a b}}{l}\left[\frac{\phi^{2}}{2}+\frac{W(\alpha)}{\alpha^{3}} \phi^{3}\right]
$$


Thus, the stress tensor components are

$$
\begin{aligned}
\tau_{t t} & =\frac{l}{R}\left[\frac{\mu}{8 \pi G l^{2}}+\frac{1}{l^{4}}\left(W-\frac{\alpha \beta}{3}\right)\right]+O\left(R^{-2}\right) \\
\tau_{\theta \theta} & =\frac{l}{R}\left[\frac{\mu}{16 \pi G}-\frac{1}{l^{2}}\left(W-\frac{\alpha \beta}{3}\right)\right]+O\left(R^{-2}\right) \\
\tau_{\phi \phi} & =\frac{l \sin ^{2} \theta}{R}\left[\frac{\mu}{16 \pi G}-\frac{1}{l^{2}}\left(W-\frac{\alpha \beta}{3}\right)\right]+O\left(R^{-2}\right)
\end{aligned}
$$

The stress tensor of the dual field theory can be put in a similar form as for the SchwarzschildAdS black hole:

$$
\left\langle\tau_{a b}^{\text {dual }}\right\rangle=\frac{3 \mu}{16 \pi G l^{2}} \delta_{a}^{0} \delta_{b}^{0}+\frac{\gamma_{a b}}{l^{2}}\left[\frac{\mu}{16 \pi G}-\frac{1}{l^{2}}\left(W(\alpha)-\frac{\alpha \beta}{3}\right)\right]
$$

The trace can be easily computed and we get

$$
\left\langle\tau^{\text {dual }}\right\rangle=-\frac{3}{l^{4}}\left[W(\alpha)-\frac{\alpha \beta}{3}\right]
$$

Unlike the Schwarzschild-AdS black hole, for the hairy black holes there are two different types of boundary conditions, namely that preserve or not the conformal symmetry. As expected, when the conformal symmetry is preserved $W=C \alpha^{3}$ the trace of the dual stress tensor vanishes $\left\langle\tau^{\text {dual }}\right\rangle=0$.

A similar, but more complicated, procedure can be applied for the logarithmic branch. The action (3.2) has a new contribution (3.25) that cancel the logarithmic divergence, and the new regularized quasilocal stress tensor is

$$
\tau_{a b}=-\frac{1}{\kappa}\left(K_{a b}-h_{a b} K+\frac{2}{l} h_{a b}-l G_{a b}\right)-\frac{h_{a b}}{l}\left[\frac{\phi^{2}}{2}+\frac{\phi^{3}}{\alpha^{3}}\left(W-\frac{\alpha \gamma}{3}\right)+\frac{\phi^{3} \gamma}{3 \alpha^{2}} \ln \left(\frac{\alpha}{\phi}\right)\right]
$$

with the following components

$$
\begin{aligned}
\tau_{t t} & =\frac{l}{R}\left[\frac{\mu}{8 \pi G l^{2}}+\frac{1}{l^{4}}\left(W-\frac{\alpha \beta}{3}-\frac{\alpha \gamma}{9}\right)\right]+O\left[\frac{(\ln R)^{3}}{R^{2}}\right] \\
\tau_{\theta \theta} & =\frac{l}{R}\left[\frac{\mu}{16 \pi G}-\frac{1}{l^{2}}\left(W-\frac{\alpha \beta}{3}-\frac{\alpha \gamma}{9}\right)\right]+O\left[\frac{(\ln R)^{3}}{R^{2}}\right] \\
\tau_{\phi \phi} & =\frac{l \sin ^{2} \theta}{R}\left[\frac{\mu}{16 \pi G}-\frac{1}{l^{2}}\left(W-\frac{\alpha \beta}{3}-\frac{\alpha \gamma}{9}\right)\right]+O\left[\frac{(\ln R)^{3}}{R^{2}}\right]
\end{aligned}
$$

and so the stress tensor of the dual field theory becomes

$$
\left\langle\tau_{a b}^{\text {dual }}\right\rangle=\frac{3 \mu}{16 \pi G l^{2}} \delta_{a}^{0} \delta_{b}^{0}+\frac{\gamma_{a b}}{l^{2}}\left[\frac{\mu}{16 \pi G}-\frac{1}{l^{2}}\left(W(\alpha)-\frac{\alpha \beta}{3}-\frac{\alpha \gamma}{9}\right)\right]
$$

Its trace is

$$
\left\langle\tau^{\text {dual }}\right\rangle=-\frac{3}{l^{4}}\left(W-\frac{\alpha \beta}{3}-\frac{\alpha \gamma}{9}\right)
$$

and, as expected, it vanishes for the boundary conditions that preserve the conformal symmetry:

$$
\left\langle\tau^{\text {dual }}\right\rangle=0 \Rightarrow \gamma=-3 l^{2} \lambda \alpha^{2} ; \quad W(\alpha)=\alpha^{3}\left[C+l^{2} \lambda \ln \alpha\right]
$$




\section{$5 \quad$ Hamiltonian mass and holographic mass}

In AdS spacetime there exist different methods of computing the gravitational mass and a comparison between them is going to be useful - for Dirichlet boundary conditions this was done in great detail in [46] and up to some ambiguities related to constant boundary terms, the Hamiltonian formalism, AMD mass, and holographic method produce the same result. Though, as was pointed out in [14], when the conformal symmetry is broken in the boundary the AMD mass is not the correct physical mass and one should compute the Hamiltonian mass of the system. In this section we provide details of computing the Hamiltonian mass and show that it matches the holographic mass even when the conformal symmetry in the boundary is broken.

\subsection{Hamiltonian formalism}

We consider the Regge-Teitelboim approach [47] to compute the mass of static scalar hairy asymptotically locally AdS spacetimes. A summary of this method is provided below. We are considering the action (2.5) for which the Hamiltonian constraints $\mathcal{H}_{\perp}$ and $\mathcal{H}_{i}$, with $i=1,2,3$, contain contributions from the gravitational term and from the matter sector that in this case corresponds to a minimally coupled scalar field with a self-interaction potential $V(\phi)$. These constraints are functions of the canonical variables: the threedimensional metric $g_{i j}$ and the scalar field $\phi$, and their corresponding conjugate momenta $\pi^{i j}$ and $\pi_{\phi}$. The Hamiltonian constraints are given by

$$
\begin{aligned}
\mathcal{H}_{\perp}= & \frac{2 \kappa}{\sqrt{g}}\left[\pi_{i j} \pi^{i j}-\frac{1}{2}\left(\pi_{i}^{i}\right)^{2}\right]-\frac{1}{2 \kappa} \sqrt{g}^{(3)} R \\
& +\frac{1}{2}\left(\frac{\pi_{\phi}^{2}}{\sqrt{g}}+\sqrt{g} g^{i j} \phi,{ }_{i} \phi, j\right)+\sqrt{g} V(\phi) \\
\mathcal{H}_{i}= & -2 \pi_{i \mid j}^{j}+\pi_{\phi} \phi, i
\end{aligned}
$$

The three-dimensional metric $g_{i j}$ can be recognized from the line element written in its ADM form

$$
d s^{2}=-\left(N^{\perp}\right)^{2} d t^{2}+g_{i j}\left(d x^{i}+N^{i} d t\right)\left(d x^{j}+N^{j} d t\right)
$$

and, $g,{ }^{(3)} R$ and vertical bar $\mid$ denote the determinant, the scalar curvature, and the covariant derivative associated to the spatial metric, respectively.

The canonical generator of an asymptotic symmetry defined by the vector $\xi=\left(\xi^{\perp}, \xi^{i}\right)$ is a linear combination of the constraints $\mathcal{H}_{\perp}, \mathcal{H}_{i}$ plus a surface term $Q[\xi]$

$$
H[\xi]=\int_{\partial \mathcal{M}} d^{3} x\left(\xi^{\perp} \mathcal{H}_{\perp}+\xi^{i} \mathcal{H}_{i}\right)+Q[\xi]
$$

$Q[\xi]$ is chosen in order to cancel out the surface terms coming from the variation of the generator with respect to the canonical variables. In this way, the generator $H[\xi]$ possesses well-defined functional derivatives [47]. The general form of $Q[\xi]$ for the generator (5.4) [12] 
is given by

$$
\begin{aligned}
\delta Q[\xi]= & \oint d^{2} S_{l}\left[\frac{G^{i j k l}}{2 \kappa}\left(\xi^{\perp} \delta g_{i j \mid k}-\xi_{, k}^{\perp} \delta g_{i j}\right)+2 \xi_{k} \delta \pi^{k l}\right. \\
& \left.+\left(2 \xi^{k} \pi^{j l}-\xi^{l} \pi^{j k}\right) \delta g_{j k}-\left(\sqrt{g} \xi^{\perp} g^{l j} \phi, j+\xi^{l} \pi_{\phi}\right) \delta \phi\right]
\end{aligned}
$$

where

$$
G^{i j k l} \equiv \frac{1}{2} \sqrt{g}\left(g^{i k} g^{j l}+g^{i l} g^{j k}-2 g^{i j} g^{k l}\right)
$$

The normal and tangential components of the allowed deformation $\left(\xi^{\perp}, \xi^{i}\right)$ are related with the spacetime components $\left(\xi^{\perp},{ }^{(3)} \xi^{i}\right)$ in the following way

$$
\xi^{\perp}=N^{\perp} \xi^{t}, \quad \xi^{i}={ }^{(3)} \xi^{i}+N^{i} \xi^{t}
$$

The following step is to note that the Hamiltonian generator (5.4) reduces to the surface term $Q[\xi]$ when the constraints hold. Thus, the value of the generators - the conserved charges associated the the asymptotic symmetries - are just given by $Q[\xi]$. Since the charges are defined by a surface term at the boundary, they need just the behaviour of the canonical variables and symmetries close to the boundary. i.e. their asymptotic behavior. Thus, the charges obtained from the Hamiltonian method are appropriate for a holographic interpretation. Additionally, one can remark that the canonical generators provide the charges for all the solutions sharing the same asymptotic behaviour.

We focus now in the static case. By definition there is a timelike Killing vector $\partial_{t}$, and the corresponding conserved charge associated with this symmetry - time translationis from first principles the mass $M$. In the static case all the momenta vanish and the expression (5.5), evaluated for $\xi=\partial_{t}$, reduces to

$$
\delta M \equiv \delta Q\left[\partial_{t}\right]=\oint d^{2} S_{l}\left[\frac{G^{i j k l}}{2 \kappa}\left(\xi^{\perp} \delta g_{i j \mid k}-\xi_{, k}^{\perp} \delta g_{i j}\right)-\sqrt{g} \xi^{\perp} g^{l j} \phi, j \delta \phi\right]
$$

We note an explicit contribution of the scalar field in the mass that, in general, yields a non-vanishing amount. In order to achieve a better understanding of this contribution, it is convenient to separate it from the usual gravitational contribution by writing $\delta M$ as

$$
\delta M=\delta M_{G}+\delta M_{\phi}
$$

where

$$
\delta M_{G}=\oint d^{2} S_{l} \frac{G^{i j k l}}{2 \kappa}\left(\xi^{\perp} \delta g_{i j \mid k}-\xi_{, k}^{\perp} \delta g_{i j}\right)
$$

and

$$
\delta M_{\phi}=-\oint d^{2} S_{l} \sqrt{g} \xi^{\perp} g^{l j} \phi,{ }_{j} \delta \phi
$$

As mentioned before, the variation of the mass, given by surface integral (5.8), needs just the asymptotic behavior of the canonical variables and symmetries. However, this variation usually requires more information to be integrated, and boundary conditions must be imposed. The necessity of boundary conditions is expected from physical grounds, 
since the mass of a system is well defined after imposing suitable boundary conditions. The effect of a slow fall-off scalar field on the mass of asymptotically hairy spacetimes have been studied in $[7,9,12]$ using the Hamiltonian formalism described above. Other approaches and methods $[8,10,13,48,49]$ have confirmed this effect.

One step further was made in [14] where the computation of the mass of these hairy configurations was done by considering the additional information provided by the remaining field equations. For this work, we focus on the analysis on the class of potentials having a mass term corresponding to the conformal mass $m^{2}=-2 l^{-2}$ in four dimensions. For completeness and because the boundary conditions and the way the divergences cancel in the construction of the Hamiltonian mass provide helpful intuition for constructing the counterterms, in the next subsection we present the details of the analysis of [14].

\subsection{Non-logarithmic and logarithmic branches for $m^{2}=-2 / l^{2}$}

Expanding the potential as a power series around $\phi=0$, it was shown [12] the absence of logarithmic branches in the asymptotically behavior of the metric and scalar field, provided the series does not contain a cubic term. This set of asymptotic conditions accommodates exact scalar black hole solutions $[18,31,50,51]$ whose asymptotic behavior belong the chosen one. The fall-off of the scalar field and metric at infinity was obtained in section 2 .

Now, we evaluate the general expressions (5.10) and (5.11) for static configurations, using the above asymptotic conditions. We consider a boundary located at $r=\infty$. Integrating the 'angular coordinates', we obtain the gravitational contribution

$$
\delta M_{G}=\frac{\sigma_{k}}{\kappa}[r \delta a+l \delta b+O(1 / r)]
$$

and the contribution from the scalar field

$$
\delta M_{\phi}=\frac{\sigma_{k}}{l^{2}}[r \alpha \delta \alpha+\alpha \delta \beta+2 \beta \delta \alpha+O(1 / r)]
$$

By adding both contributions we have the variation of the mass

$$
\delta M=\frac{\sigma_{k}}{\kappa l^{2}}\left[r\left(l^{2} \delta a+\kappa \alpha \delta \alpha\right)+l^{3} \delta b+\kappa(\alpha \delta \beta+2 \beta \delta \alpha)+O(1 / r)\right]
$$

It is important to remind that this expression for $\delta M$ is meaningful only in the case of vanishing constraints. In the static case, there is a single nontrivial constraint, $H_{\perp}=0$, which for the asymptotic conditions displayed above yields

$$
\frac{k+a}{\kappa}+\frac{\alpha^{2}}{2 l^{2}}=0
$$

The linear divergent piece in (5.14) is removed by replacing (5.15) into (5.14). Then, the asymptotic variation of the mass becomes finite

$$
\delta M=\frac{\sigma_{k}}{\kappa l^{2}}\left[l^{3} \delta b+\kappa(\alpha \delta \beta+2 \beta \delta \alpha)\right]
$$

In order to integrate the variations in (5.16) boundary conditions on the scalar field are necessary. In particular, the integration of (5.16) requires a functional relation between $\alpha$ and $\beta$. If we define $\beta=d W(\alpha) / d \alpha$, the mass of the spacetime is given by

$$
M=\sigma_{k}\left[\frac{l b}{\kappa}+\frac{1}{l^{2}}\left(\alpha \frac{d W(\alpha)}{d \alpha}+W(\alpha)\right)\right]
$$


We note that the mass in (5.17) is defined up to a constant without variation. This constant is set to be zero in order to fix a vanishing mass for the locally AdS spacetime because in four dimensions there is no Casimir energy.

To obtain the logarithmic branch, it is necessary to use the self-interaction potential (2.18) so that the fall-off of the scalar field to be considered is (2.19). The Hamiltonian constraint $H_{\perp}=0$ is satisfied if (5.15) and

$$
\frac{l c}{\kappa}-4 \alpha^{3} \lambda=0
$$

are fulfilled.

Now, we evaluate (5.10) and (5.11). In this case we find

$$
\delta M_{G}=\left\{\frac{l \delta b}{\kappa}+\frac{\delta a}{\kappa} r+\frac{l \delta c}{\kappa} \ln (r)+O\left(\frac{\ln (r)^{2}}{r}\right)\right\} \sigma_{k}
$$

and

$$
\begin{aligned}
\delta M_{\phi}= & {\left[\frac{\alpha \delta \beta+2 \beta \delta \alpha+3 \alpha^{2} l^{2} \lambda \delta \alpha}{l^{2}}+r \frac{\alpha \delta \alpha}{l^{2}}\right.} \\
& \left.-12 \lambda \alpha^{2} \delta \alpha \ln (r)+O\left(\frac{\ln (r)^{2}}{r}\right)\right] \sigma_{k}
\end{aligned}
$$

Both contributions contain linear and logarithmic divergences. Adding (5.19) and (5.20), the linear divergence cancels out by virtue of (5.15) and the logarithmic divergence vanishes by considering (5.18). Thus, we obtain a finite expression for the variation of the mass,

$$
\delta M=\left[\frac{l \delta b}{\kappa}+\frac{\alpha \delta \beta+2 \beta \delta \alpha+3 \alpha^{2} l^{2} \lambda \delta \alpha}{l^{2}}\right] \sigma_{k}
$$

Again, we need a boundary condition, a functional relation between $\alpha$ and $\beta$, in order to integrate $\delta M$. We consider the general relation $\beta=\frac{d W}{d \alpha}$, so that the Hamiltonian mass is given by

$$
M=\left[\frac{l b}{\kappa}+\frac{1}{l^{2}}\left(\alpha \frac{d W}{d \alpha}+W(\alpha)+\alpha^{3} l^{2} \lambda\right)\right] \sigma_{k}
$$

The mass can be related with the first subleading term of $g_{t t}$ by using (2.22). Thus the mass can be written as

$$
M=\left[\frac{\mu}{\kappa}+\frac{1}{l^{2}}\left(W(\alpha)-\frac{1}{3} \alpha \frac{d W}{d \alpha}+\frac{1}{3} \alpha^{3} l^{2} \lambda\right)\right] \sigma_{k}
$$

Therefore, the expression $M=\mu \sigma_{k} \kappa^{-1}$ is obtained only for $\alpha=0$ or

$$
W(\alpha)=\alpha^{3}\left[C+l^{2} \lambda \ln (\alpha)\right]
$$

which correspond to AdS invariant boundary conditions [12]. 


\subsection{Holographic mass matches Hamiltonian mass}

Armed with the Brown-York formalism supplemented with counterterms, one can obtain the energy of a hairy black holes. The boundary metric can be written, at least locally, in ADM-like form. Provided the boundary geometry has an isometry generated by the Killing vector $\xi^{a}=\left(\partial_{t}\right)^{a}$, the energy is, as usual, the conserved charge.

Concretely, we are going to use the coordinates $\left(t, r, \Sigma_{k}\right)$ with the metric (2.9) and the foliation (4.2) parametrized as

$$
d \Sigma_{k}^{2}=\frac{d y^{2}}{1-k y^{2}}+\left(1-k y^{2}\right) d \phi^{2}
$$

The energy

$$
E=\int d \sigma^{i} \tau_{i j} \xi^{j}=\int d y d \phi S u^{i} \tau_{i j} \xi^{j}
$$

is associated with the surface $t=$ constant, for which the induced metric is

$$
d s^{2}=\sigma_{i j} d x^{i} d x^{j}=S d \Sigma_{k}^{2}
$$

with the normal vector $u^{a}=N^{-1 / 2}\left(\partial_{t}\right)^{a}$.

For the non-logarithmic branch, using the quasilocal stress tensor (4.9), one obtains

$$
E=\sigma_{k}\left[\frac{\mu}{\kappa}+\frac{1}{l^{2}}\left(W-\frac{\alpha}{3} \frac{d W}{d \alpha}\right)\right]
$$

With a similar computation for the logarithmic branch, but with the quasilocal stress tensor (4.13), we obtain the following energy of the hairy black hole:

$$
E=\sigma_{k}\left[\frac{\mu}{\kappa}+\frac{1}{l^{2}}\left(W-\frac{1}{3} \alpha \frac{d W}{d \alpha}-\frac{\alpha \gamma}{9}\right)\right]=\sigma_{k}\left[\frac{\mu}{\kappa}+\frac{1}{l^{2}}\left(W-\frac{1}{3} \alpha \frac{d W}{d \alpha}-\frac{\alpha^{3} C_{\gamma}}{9}\right)\right]
$$

This shows perfect agreement with the Hamiltonian mass even if the conformal symmetry is broken in the boundary - with both methods it is possible to obtain a finite energy in this case and the corresponding results match. However, the AMD prescription [28, 29] for computing the mass of a hairy spacetime is not suitable when the scalar field breaks the asymptotic anti-de Sitter invariance [14].

\subsection{Exact hairy solutions and triple-trace deformations}

As a concrete example, we discuss the boundary conditions and some holographic properties of the exact solutions of $[18,31]$. We consider the following scalar potential, which for some particular values of the parameter $\Upsilon$ it becomes the one of a truncation of $\omega$-deformed gauged $\mathcal{N}=8$ supergravity $[31,33,52]$ :

$$
\begin{aligned}
V(\phi)= & \frac{\Lambda\left(\nu^{2}-4\right)}{6 \kappa \nu^{2}}\left[\frac{\nu-1}{\nu+2} e^{-\phi l_{\nu}(\nu+1)}+\frac{\nu+1}{\nu-2} e^{\phi l_{\nu}(\nu-1)}+4 \frac{\nu^{2}-1}{\nu^{2}-4} e^{-\phi l_{\nu}}\right] \\
& +\frac{\Upsilon}{\kappa \nu^{2}}\left[\frac{\nu-1}{\nu+2} \sinh \phi l_{\nu}(\nu+1)-\frac{\nu+1}{\nu-2} \sinh \phi l_{\nu}(\nu-1)+4 \frac{\nu^{2}-1}{\nu^{2}-4} \sinh \phi l_{\nu}\right]
\end{aligned}
$$


Using the metric ansatz (3.8), the equations of motion can be integrated for the conformal factor $[17-19,53]$ :

$$
\Omega(x)=\frac{\nu^{2} x^{\nu-1}}{\eta^{2}\left(x^{\nu}-1\right)^{2}}
$$

where $\Upsilon, \nu, \kappa$ and $\Lambda=-3 l^{-2}$ are parameters of the potential and $\eta$ is an integration constant. All of them characterize the hairy solution. With this choice of the conformal factor, it is straightforward to obtain the expressions for the scalar field

$$
\phi(x)=l_{\nu}^{-1} \ln x
$$

and metric function

$$
f(x)=\frac{1}{l^{2}}+\Upsilon\left[\frac{1}{\nu^{2}-4}-\frac{x^{2}}{\nu^{2}}\left(1+\frac{x^{-\nu}}{\nu-2}-\frac{x^{\nu}}{\nu+2}\right)\right]+\frac{x}{\Omega(x)}
$$

where $l_{\nu}^{-1}=\sqrt{\left(\nu^{2}-1\right) / 2 \kappa}$.

We would like to point out that this potential is symmetric under $\nu \rightarrow-\nu$. For $x=1$, which corresponds to the boundary, we can show that the theory has a standard AdS vacuum $2 \kappa V(\phi=0)=2 \Lambda$. In the limit $\nu=1$, one gets $l_{\nu} \rightarrow \infty$ and $\phi \rightarrow 0$ so that the Schwarzschild-AdS black hole is smoothly obtained.

To compare with the results presented in the previous section, we should work with the canonical coordinates of AdS. Let us discuss the branch $x \in(1, \infty)$ for which the scalar field is positively defined. We change the $r$-coordinate so that the function in front of the transversal section, $d \Sigma_{k}$, has the following fall-off:

$$
\Omega(x)=r^{2}+O\left(r^{-3}\right)
$$

This choice is motivated by the fact that the term $O\left(r^{-2}\right)$ generates a lineal term in the fall-off of $\Omega$. The first three subleading terms are

$$
x=1+\frac{1}{\eta r}+\frac{m}{r^{3}}+\frac{n}{r^{4}}+\frac{p}{r^{5}}+O\left(r^{-6}\right)
$$

and they can be computed by considering the expansion around $r=\infty$ :

$\Omega(x)=r^{2}-\frac{24 m \eta^{3}+\nu^{2}-1}{12 \eta^{2}}-\frac{24 n \eta^{4}-\nu^{2}+1}{12 \eta^{3} r}+\frac{720 m^{2} \eta^{6}-480 p \eta^{5}+\nu^{4}-20 \nu^{2}+19}{240 \eta^{4} r^{2}}+O\left(r^{-3}\right)$

After a straightforward computation we obtain

$$
x=1+\frac{1}{\eta r}-\frac{\left(\nu^{2}-1\right)}{24 \eta^{3} r^{3}}\left[1-\frac{1}{\eta r}-\frac{9\left(\nu^{2}-9\right)}{80 \eta^{2} r^{2}}\right]+O\left(r^{-6}\right)
$$

and the following asymptotic expansions for the metric functions:

$$
\begin{aligned}
-g_{t t} & =f(x) \Omega(x)=\frac{r^{2}}{l^{2}}+1+\frac{\Upsilon+3 \eta^{2}}{3 \eta^{3} r}+O\left(r^{-3}\right) \\
g_{r r} & =\frac{\Omega(x) \eta^{2}}{f(x)}\left(\frac{d x}{d r}\right)=\frac{l^{2}}{r^{2}}-\frac{l^{4}}{r^{4}}-\frac{l^{2}\left(\nu^{2}-1\right)}{4 \eta^{2} r^{4}}-\frac{l^{2}\left(3 \eta^{2} l^{2}+\Upsilon l^{2}-\nu^{2}+1\right)}{3 \eta^{3} r^{5}}+O\left(r^{-6}\right)
\end{aligned}
$$


The asymptotic expansion of the scalar field becomes in these coordinates

$$
\phi(x)=l_{\nu}^{-1} \ln x=\frac{1}{l_{\nu} \eta r}-\frac{1}{2 l_{\nu} \eta^{2} r^{2}}-\frac{\nu^{2}-9}{24 \eta^{3} r^{3}}+O\left(r^{-4}\right)
$$

and then, in the standard notation, we obtain $\alpha=1 / l_{\nu} \eta, \beta=-1 / 2 l_{\nu} \eta^{2}$. Both modes are normalizable and, since $\beta=C \alpha^{2}$ with $C=-l_{\nu} / 2$, the conformal symmetry in the boundary is preserved. Now, we can easily compute the Hamiltonian mass of the system as was proposed in [14]

$$
M=\sigma\left[\frac{\mu}{\kappa}+\frac{1}{l^{2}}\left(W-\frac{\alpha}{3} \frac{d W}{d \alpha}\right)\right]
$$

and by considering $W=-l_{\nu} \alpha^{3} / 6, \sigma=4 \pi$, and $l_{\nu}^{-1}=\sqrt{\left(\nu^{2}-1\right) / 2 \kappa}$ we obtain

$$
M=-\frac{\sigma}{\kappa}\left(\frac{3 \eta^{2}+\Upsilon}{3 \eta^{3}}\right)
$$

that matches the holographic mass.

Let us end up this subsection with the interpretation of these hairy solutions within AdS/CFT duality. That is, since $W=-l_{\nu} \alpha^{3} / 6$, they correspond to adding a triple trace deformation to the boundary action as in (2.32) (similar examples can be found in $[10,54]$ ):

$$
I_{\mathrm{CFT}} \rightarrow I_{\mathrm{CFT}}+\frac{l_{\nu}}{6} \int d^{3} x \mathcal{O}^{3}
$$

For different hairy black holes, which are characterized by the hairy parameter $\nu$, the relation between $\alpha$ and $\beta$ does not change and so there are triple trace deformations, but with different couplings $l_{\nu} / 6$.

\section{Conclusions}

Since the paper is self-contained and each section contains detailed computations and interpretations, we would like only to present some general conclusions and possible future directions.

The counterterm method [3], which was obtained in the context of AdS/CFT duality, is by now a textbook example of regularizing the gravitational action. Initially it was proposed for asymptotically AdS solutions $[22,55,56]$ and then it was generalized to asymptotically flat solutions [57-64] and even dS solutions [65-68], though in the last two cases it is fair to say that there is no valid holographic interpretation generally accepted. Interestingly, this method provides the quasilocal stress tensor and conserved charges in a very similar way with the well understood holography of asymptotically AdS spacetimes.

When the theory contains scalar fields, there is a diversity of mixed boundary conditions that can be imposed, in particular boundary conditions that break the conformal symmetry of the boundary. The 'holographic renormalization' method $[21,22,55,56]$ that uses the Fefferman-Graham expansion was generalized for the mixed boundary conditions that correspond to the non-logarithmic branch of solutions in [24]. 
In this work we have constructed explicit covariant counterterms that are similar with the ones proposed by Balasubramanian and Kraus [3] and generalized this method for theories (moduli potentials) that contain also the logarithmic branch of solutions. To construct these counterterms we were guided by the Hamiltonian method that provides the correct boundary conditions (in particular, the fall-off of the scalar field) so that the conserved charges are finite. We did also check that the variational principle for the gravitational action is well-posed. It may then not be surprising that the holographic mass matches the Hamiltonian mass for all the boundary conditions. However, when comparing with AMD formalism there is a drastic change when the boundary conditions do not preseve the conformal symmetry and, as was shown in [14], the AMD mass is not suitable for this case.

As future directions we would like to consider counterterms for other conformal masses of the scalar field and for theories in higher dimensions. It will be useful, if possible, to provide a general algorithm for constructing the counterterms by using the Hamiltonian method - it is not at all clear if that is possible for gravity solutions that are asymptotically dS. For the extremal black holes, there exists a different method to compute the conserved charges, the entropy function formalism proposed by Sen [69-71] (for spinning black holes it was generalized in [72] and in the context of AdS/CFT duality, see e.g. [73-77]). However, this method provides the charges by using the near horizon geometry data and, when there is a non-trivial RG flow in theories with scalars turned on, it will be interesting to compare the conserved charges computed at the horizon with the ones obtained at the boundary by the counterterm method. The counterterm method was already used in [78] to study the phase diagram of a general class of hairy black holes with spherical horizon geometry and we also plan to study the phase transitions for the case $k=0$ [79] when a 'hairy' AdS soliton can be constructed.

A different perspective, which naturally arises when scalar fields and gravity interact, is the classical issue of hairy black holes. Indeed, it was very early shown that in asymptotically flat spacetimes and when the scalar field potential is convex, the only spherically symmetric black hole is the Schwarzschild solution [80, 81], which was later generalized to non-negative self interactions [82, 83], for a recent review see [84]. These no-hair theorems were not expected to hold when, asymptotically, there is a non-trivial cosmological constant. The numerical existence of asymptotically AdS black holes has been verified in a number of papers $[10,85,86]$ (a number exact hairy black holes has been found when the scalar field mass is $\left.m^{2}=-2 l^{-2}[18,31,50,51,87-90]\right)$. Some of these black holes are linearly stable [85, 91]. Another interesting direction is on boson star solutions and the relation with the instabilities of some AdS solutions (and AdS itself) [92-96].

We hope to report in the near future some progress in these directions.

\section{Acknowledgments}

DA would like to thank Stefan Theisen for interesting discussions on conformal anomalies. DA and DC acknowledge the hospitality of the Albert Einstein Institute in Potsdam during the last stages of this research. This work has been done with partial support from the Fondecyt grants 11121187, 1120446, 1121031,1130658, and 1141073 and by the 
Newton-Picarte Grants DPI20140053 and DPI20140115. D. C. thanks CONICYT for a Ph.D. scholarship. The Centro de Estudios Científicos (CECs) is funded by the Chilean Government through the Centers of Excellence Base Financing Program of Conicyt.

Open Access. This article is distributed under the terms of the Creative Commons Attribution License (CC-BY 4.0), which permits any use, distribution and reproduction in any medium, provided the original author(s) and source are credited.

\section{References}

[1] J.M. Maldacena, The Large- $N$ limit of superconformal field theories and supergravity, [Adv. Theor. Math. Phys. 2 (1998) 231] [Int. J. Theor. Phys. 38 (1999) 1113] [hep-th/9711200] [INSPIRE].

[2] M. Henningson and K. Skenderis, The Holographic Weyl anomaly, JHEP 07 (1998) 023 [hep-th/9806087] [INSPIRE].

[3] V. Balasubramanian and P. Kraus, A Stress tensor for Anti-de Sitter gravity, Commun. Math. Phys. 208 (1999) 413 [hep-th/9902121] [INSPIRE].

[4] A. Ishibashi and R.M. Wald, Dynamics in nonglobally hyperbolic static space-times. 3. Anti-de Sitter space-time, Class. Quant. Grav. 21 (2004) 2981 [hep-th/0402184] [INSPIRE].

[5] P. Breitenlohner and D.Z. Freedman, Positive Energy in anti-de Sitter Backgrounds and Gauged Extended Supergravity, Phys. Lett. B 115 (1982) 197 [InSPIRE].

[6] P. Breitenlohner and D.Z. Freedman, Stability in Gauged Extended Supergravity, Annals Phys. 144 (1982) 249 [INSPIRE].

[7] M. Henneaux, C. Martínez, R. Troncoso and J. Zanelli, Black holes and asymptotics of 2+1 gravity coupled to a scalar field, Phys. Rev. D 65 (2002) 104007 [hep-th/0201170] [INSPIRE].

[8] G. Barnich, Conserved charges in gravitational theories: contribution from scalar fields, gr-qc/0211031 [INSPIRE].

[9] M. Henneaux, C. Martínez, R. Troncoso and J. Zanelli, Asymptotically anti-de Sitter spacetimes and scalar fields with a logarithmic branch, Phys. Rev. D 70 (2004) 044034 [hep-th/0404236] [INSPIRE].

[10] T. Hertog and K. Maeda, Black holes with scalar hair and asymptotics in $N=8$ supergravity, JHEP 07 (2004) 051 [hep-th/0404261] [INSPIRE].

[11] T. Hertog and G.T. Horowitz, Designer gravity and field theory effective potentials, Phys. Rev. Lett. 94 (2005) 221301 [hep-th/0412169] [INSPIRE].

[12] M. Henneaux, C. Martínez, R. Troncoso and J. Zanelli, Asymptotic behavior and Hamiltonian analysis of anti-de Sitter gravity coupled to scalar fields, Annals Phys. $\mathbf{3 2 2}$ (2007) 824 [hep-th/0603185] [INSPIRE].

[13] A.J. Amsel and D. Marolf, Energy Bounds in Designer Gravity, Phys. Rev. D 74 (2006) 064006 [Erratum ibid. D 75 (2007) 029901] [hep-th/0605101] [InSPIRE].

[14] A. Anabalón, D. Astefanesei and C. Martínez, Mass of asymptotically anti-de Sitter hairy spacetimes, Phys. Rev. D 91 (2015) 041501 [arXiv:1407.3296] [InSPIRE].

[15] E. Witten, Multitrace operators, boundary conditions and AdS/CFT correspondence, hep-th/0112258 [INSPIRE]. 
[16] O. Aharony, G. Gur-Ari and N. Klinghoffer, The Holographic Dictionary for $\beta$-functions of Multi-trace Coupling Constants, JHEP 05 (2015) 031 [arXiv:1501.06664] [INSPIRE].

[17] A. Acena, A. Anabalón and D. Astefanesei, Exact hairy black brane solutions in AdS $S_{5}$ and holographic RG flows, Phys. Rev. D 87 (2013) 124033 [arXiv:1211.6126] [INSPIRE].

[18] A. Aceña, A. Anabalón, D. Astefanesei and R. Mann, Hairy planar black holes in higher dimensions, JHEP 01 (2014) 153 [arXiv:1311.6065] [INSPIRE].

[19] A. Anabalón and D. Astefanesei, On attractor mechanism of AdS 4 black holes, Phys. Lett. B 727 (2013) 568 [arXiv: 1309.5863] [INSPIRE].

[20] Z.-Y. Fan and B. Chen, Exact formation of hairy planar black holes, arXiv:1512.09145 [INSPIRE].

[21] K. Skenderis, Asymptotically Anti-de Sitter space-times and their stress energy tensor, Int. J. Mod. Phys. A 16 (2001) 740 [hep-th/0010138] [INSPIRE].

[22] K. Skenderis, Lecture notes on holographic renormalization, Class. Quant. Grav. 19 (2002) 5849 [hep-th/0209067] [INSPIRE].

[23] J.D. Brown and J.W. York Jr., Quasilocal energy and conserved charges derived from the gravitational action, Phys. Rev. D 47 (1993) 1407 [gr-qc/9209012] [INSPIRE].

[24] I. Papadimitriou, Multi-Trace Deformations in AdS/CFT: Exploring the Vacuum Structure of the Deformed CFT, JHEP 05 (2007) 075 [hep-th/0703152] [INSPIRE].

[25] J. Aparicio, D. Grumiller, E. Lopez, I. Papadimitriou and S. Stricker, Bootstrapping gravity solutions, JHEP 05 (2013) 128 [arXiv:1212.3609] [INSPIRE].

[26] S. Nojiri and S.D. Odintsov, Conformal anomaly for dilaton coupled theories from AdS/CFT correspondence, Phys. Lett. B 444 (1998) 92 [hep-th/9810008] [INSPIRE].

[27] S. Nojiri, S.D. Odintsov and S. Ogushi, Finite action in d-5 gauged supergravity and dilatonic conformal anomaly for dual quantum field theory, Phys. Rev. D 62 (2000) 124002 [hep-th/0001122] [INSPIRE].

[28] A. Ashtekar and S. Das, Asymptotically Anti-de Sitter space-times: Conserved quantities, Class. Quant. Grav. 17 (2000) L17 [hep-th/9911230] [INSPIRE].

[29] A. Ashtekar and A. Magnon, Asymptotically anti-de Sitter space-times, Class. Quant. Grav. 1 (1984) L39 [INSPIRE].

[30] D.D.K. Chow and G. Compère, Dyonic AdS black holes in maximal gauged supergravity, Phys. Rev. D 89 (2014) 065003 [arXiv:1311.1204] [INSPIRE].

[31] A. Anabalón and D. Astefanesei, Black holes in $\omega$-defomed gauged $N=8$ supergravity, Phys. Lett. B 732 (2014) 137 [arXiv: 1311.7459] [INSPIRE].

[32] G. Dall'Agata, G. Inverso and M. Trigiante, Evidence for a family of $\mathrm{SO}(8)$ gauged supergravity theories, Phys. Rev. Lett. 109 (2012) 201301 [arXiv:1209.0760] [INSPIRE].

[33] J. Tarrío and O. Varela, Electric/magnetic duality and RG flows in $A d S_{4} / C F T_{3}, J H E P 01$ (2014) 071 [arXiv: 1311.2933] [INSPIRE].

[34] G. Dibitetto, A. Guarino and D. Roest, Lobotomy of Flux Compactifications, JHEP 05 (2014) 067 [arXiv: 1402.4478] [INSPIRE].

[35] A. Gallerati, H. Samtleben and M. Trigiante, The $\mathcal{N}>2$ supersymmetric AdS vacua in maximal supergravity, JHEP 12 (2014) 174 [arXiv:1410.0711] [INSPIRE]. 
[36] R. Emparan, C.V. Johnson and R.C. Myers, Surface terms as counterterms in the AdS/CFT correspondence, Phys. Rev. D 60 (1999) 104001 [hep-th/9903238] [INSPIRE].

[37] V. Balasubramanian, P. Kraus and A.E. Lawrence, Bulk versus boundary dynamics in anti-de Sitter space-time, Phys. Rev. D 59 (1999) 046003 [hep-th/9805171] [INSPIRE].

[38] V. Balasubramanian, P. Kraus, A.E. Lawrence and S.P. Trivedi, Holographic probes of anti-de Sitter space-times, Phys. Rev. D 59 (1999) 104021 [hep-th/9808017] [INSPIRE].

[39] S.S. Gubser, I.R. Klebanov and A.M. Polyakov, Gauge theory correlators from noncritical string theory, Phys. Lett. B 428 (1998) 105 [hep-th/9802109] [INSPIRE].

[40] E. Witten, Anti-de Sitter space and holography, Adv. Theor. Math. Phys. 2 (1998) 253 [hep-th/9802150] [INSPIRE].

[41] H. Lü, Y. Pang and C.N. Pope, AdS Dyonic Black Hole and its Thermodynamics, JHEP 11 (2013) 033 [arXiv:1307.6243] [INSPIRE].

[42] H. Lü, C.N. Pope and Q. Wen, Thermodynamics of AdS Black Holes in Einstein-Scalar Gravity, JHEP 03 (2015) 165 [arXiv: 1408.1514] [INSPIRE].

[43] S.S. Gubser, I.R. Klebanov and A.A. Tseytlin, String theory and classical absorption by three-branes, Nucl. Phys. B 499 (1997) 217 [hep-th/9703040] [INSPIRE].

[44] S.S. Gubser and I.R. Klebanov, Absorption by branes and Schwinger terms in the world volume theory, Phys. Lett. B 413 (1997) 41 [hep-th/9708005] [INSPIRE].

[45] R.C. Myers, Stress tensors and Casimir energies in the AdS/CFT correspondence, Phys. Rev. D 60 (1999) 046002 [hep-th/9903203] [INSPIRE].

[46] S. Hollands, A. Ishibashi and D. Marolf, Comparison between various notions of conserved charges in asymptotically AdS-spacetimes, Class. Quant. Grav. 22 (2005) 2881 [hep-th/0503045] [INSPIRE].

[47] T. Regge and C. Teitelboim, Role of Surface Integrals in the Hamiltonian Formulation of General Relativity, Annals Phys. 88 (1974) 286 [INSPIRE].

[48] M. Bañados and S. Theisen, Scale invariant hairy black holes, Phys. Rev. D 72 (2005) 064019 [hep-th/0506025] [INSPIRE].

[49] J. Gegenberg, C. Martínez and R. Troncoso, A Finite action for three-dimensional gravity with a minimally coupled scalar field, Phys. Rev. D 67 (2003) 084007 [hep-th/0301190] [INSPIRE].

[50] C. Martínez, R. Troncoso and J. Zanelli, Exact black hole solution with a minimally coupled scalar field, Phys. Rev. D 70 (2004) 084035 [hep-th/0406111] [INSPIRE].

[51] A. Anabalón, Exact Black Holes and Universality in the Backreaction of non-linear $\sigma$-models with a potential in (A)dS4, JHEP 06 (2012) 127 [arXiv: 1204.2720] [INSPIRE].

[52] A. Guarino, On new maximal supergravity and its BPS domain-walls, JHEP 02 (2014) 026 [arXiv: 1311.0785] [INSPIRE].

[53] A. Anabalón, D. Astefanesei and R. Mann, Exact asymptotically flat charged hairy black holes with a dilaton potential, JHEP 10 (2013) 184 [arXiv:1308.1693] [INSPIRE].

[54] T. Hertog and G.T. Horowitz, Towards a big crunch dual, JHEP 07 (2004) 073 [hep-th/0406134] [INSPIRE]. 
[55] S. de Haro, S.N. Solodukhin and K. Skenderis, Holographic reconstruction of space-time and renormalization in the AdS/CFT correspondence, Commun. Math. Phys. 217 (2001) 595 [hep-th/0002230] [INSPIRE].

[56] M. Bianchi, D.Z. Freedman and K. Skenderis, Holographic renormalization, Nucl. Phys. B 631 (2002) 159 [hep-th/0112119] [INSPIRE].

[57] R.B. Mann and D. Marolf, Holographic renormalization of asymptotically flat spacetimes, Class. Quant. Grav. 23 (2006) 2927 [hep-th/0511096] [INSPIRE].

[58] D. Astefanesei and E. Radu, Quasilocal formalism and black ring thermodynamics, Phys. Rev. D 73 (2006) 044014 [hep-th/0509144] [INSPIRE].

[59] R.B. Mann, D. Marolf and A. Virmani, Covariant Counterterms and Conserved Charges in Asymptotically Flat Spacetimes, Class. Quant. Grav. 23 (2006) 6357 [gr-qc/0607041] [INSPIRE].

[60] D. Astefanesei, R.B. Mann and C. Stelea, Note on counterterms in asymptotically flat spacetimes, Phys. Rev. D 75 (2007) 024007 [hep-th/0608037] [INSPIRE].

[61] D. Marolf, Asymptotic flatness, little string theory and holography, JHEP 03 (2007) 122 [hep-th/0612012] [INSPIRE].

[62] D. Astefanesei, M.J. Rodriguez and S. Theisen, Quasilocal equilibrium condition for black ring, JHEP 12 (2009) 040 [arXiv:0909.0008] [INSPIRE].

[63] D. Astefanesei, R.B. Mann, M.J. Rodriguez and C. Stelea, Quasilocal formalism and thermodynamics of asymptotically flat black objects, Class. Quant. Grav. 27 (2010) 165004 [arXiv:0909.3852] [INSPIRE].

[64] D. Astefanesei, M.J. Rodriguez and S. Theisen, Thermodynamic instability of doubly spinning black objects, JHEP 08 (2010) 046 [arXiv: 1003.2421] [INSPIRE].

[65] V. Balasubramanian, J. de Boer and D. Minic, Mass, entropy and holography in asymptotically de Sitter spaces, Phys. Rev. D 65 (2002) 123508 [hep-th/0110108] [INSPIRE].

[66] A.M. Ghezelbash and R.B. Mann, Action, mass and entropy of Schwarzschild-de Sitter black holes and the de Sitter/CFT correspondence, JHEP 01 (2002) 005 [hep-th/0111217] [INSPIRE].

[67] A.M. Ghezelbash, D. Ida, R.B. Mann and T. Shiromizu, Slicing and brane dependence of the (A)dS/CFT correspondence, Phys. Lett. B 535 (2002) 315 [hep-th/0201004] [INSPIRE].

[68] D. Astefanesei, R.B. Mann and E. Radu, Reissner-Nordstrom-de Sitter black hole, planar coordinates and dS/CFT, JHEP 01 (2004) 029 [hep-th/0310273] [INSPIRE].

[69] A. Sen, Entropy function for heterotic black holes, JHEP 03 (2006) 008 [hep-th/0508042] [INSPIRE].

[70] A. Sen, Black hole entropy function and the attractor mechanism in higher derivative gravity, JHEP 09 (2005) 038 [hep-th/0506177] [INSPIRE].

[71] A. Sen, Black Hole Entropy Function, Attractors and Precision Counting of Microstates, Gen. Rel. Grav. 40 (2008) 2249 [arXiv:0708.1270] [InSPIRE].

[72] D. Astefanesei, K. Goldstein, R.P. Jena, A. Sen and S.P. Trivedi, Rotating attractors, JHEP 10 (2006) 058 [hep-th/0606244] [INSPIRE].

[73] D. Astefanesei, N. Banerjee and S. Dutta, (Un)attractor black holes in higher derivative AdS gravity, JHEP 11 (2008) 070 [arXiv:0806.1334] [INSPIRE]. 
[74] D. Astefanesei, H. Nastase, H. Yavartanoo and S. Yun, Moduli flow and non-supersymmetric AdS attractors, JHEP 04 (2008) 074 [arXiv:0711.0036] [INSPIRE].

[75] J.F. Morales and H. Samtleben, Entropy function and attractors for AdS black holes, JHEP 10 (2006) 074 [hep-th/0608044] [INSPIRE].

[76] D. Astefanesei, N. Banerjee and S. Dutta, Moduli and electromagnetic black brane holography, JHEP 02 (2011) 021 [arXiv: 1008.3852] [INSPIRE].

[77] D. Astefanesei, N. Banerjee and S. Dutta, Near horizon data and physical charges of extremal AdS black holes, Nucl. Phys. B 853 (2011) 63 [arXiv:1104.4121] [INSPIRE].

[78] A. Anabalón, D. Astefanesei and D. Choque, On the thermodynamics of hairy black holes, Phys. Lett. B 743 (2015) 154 [arXiv: 1501.04252] [INSPIRE].

[79] A. Anabalón, D. Astefanesei and D. Choque, in preparation.

[80] J.D. Bekenstein, Transcendence of the law of baryon-number conservation in black hole physics, Phys. Rev. Lett. 28 (1972) 452 [InSPIRE].

[81] J.D. Bekenstein, Nonexistence of baryon number for black holes. II, Phys. Rev. D 5 (1972) 2403 [INSPIRE].

[82] M. Heusler, A No hair theorem for selfgravitating nonlinear $\sigma$-models, J. Math. Phys. 33 (1992) 3497 [INSPIRE].

[83] D. Sudarsky, A Simple proof of a no hair theorem in Einstein Higgs theory, Class. Quant. Grav. 12 (1995) 579 [INSPIRE].

[84] C.A.R. Herdeiro and E. Radu, Asymptotically flat black holes with scalar hair: a review, Int. J. Mod. Phys. D 24 (2015) 1542014 [arXiv: 1504.08209] [INSPIRE].

[85] T. Torii, K. Maeda and M. Narita, Scalar hair on the black hole in asymptotically anti-de Sitter space-time, Phys. Rev. D 64 (2001) 044007 [INSPIRE].

[86] D. Sudarsky and J.A. Gonzalez, On black hole scalar hair in asymptotically anti-de Sitter space-times, Phys. Rev. D 67 (2003) 024038 [gr-qc/0207069] [INSPIRE].

[87] T. Kolyvaris, G. Koutsoumbas, E. Papantonopoulos and G. Siopsis, A New Class of Exact Hairy Black Hole Solutions, Gen. Rel. Grav. 43 (2011) 163 [arXiv:0911.1711] [INSPIRE].

[88] A. Anabalón, F. Canfora, A. Giacomini and J. Oliva, Black Holes with Primary Hair in gauged $N=8$ Supergravity, JHEP 06 (2012) 010 [arXiv:1203.6627] [INSPIRE].

[89] P.A. González, E. Papantonopoulos, J. Saavedra and Y. Vásquez, Four-Dimensional Asymptotically AdS Black Holes with Scalar Hair, JHEP 12 (2013) 021 [arXiv:1309.2161] [INSPIRE].

[90] X.-H. Feng, H. Lü and Q. Wen, Scalar Hairy Black Holes in General Dimensions, Phys. Rev. D 89 (2014) 044014 [arXiv: 1312.5374] [INSPIRE].

[91] A. Anabalón, D. Astefanesei and J. Oliva, Hairy Black Hole Stability in AdS, Quantum Mechanics on the Half-Line and Holography, JHEP 10 (2015) 068 [arXiv:1507.05520] [INSPIRE].

[92] D. Astefanesei and E. Radu, Boson stars with negative cosmological constant, Nucl. Phys. B 665 (2003) 594 [gr-qc/0309131] [INSPIRE].

[93] P. Bizon and A. Rostworowski, On weakly turbulent instability of anti-de Sitter space, Phys. Rev. Lett. 107 (2011) 031102 [arXiv:1104.3702] [INSPIRE]. 
[94] A. Buchel, S.L. Liebling and L. Lehner, Boson stars in AdS spacetime, Phys. Rev. D 87 (2013) 123006 [arXiv: 1304.4166] [INSPIRE].

[95] D. Astefanesei and E. Radu, Rotating boson stars in (2+1)-dimenmsions, Phys. Lett. B 587 (2004) 7 [gr-qc/0310135] [inSPIRE].

[96] A. Buchel, AdS boson stars in string theory, arXiv:1510.08415 [INSPIRE]. 